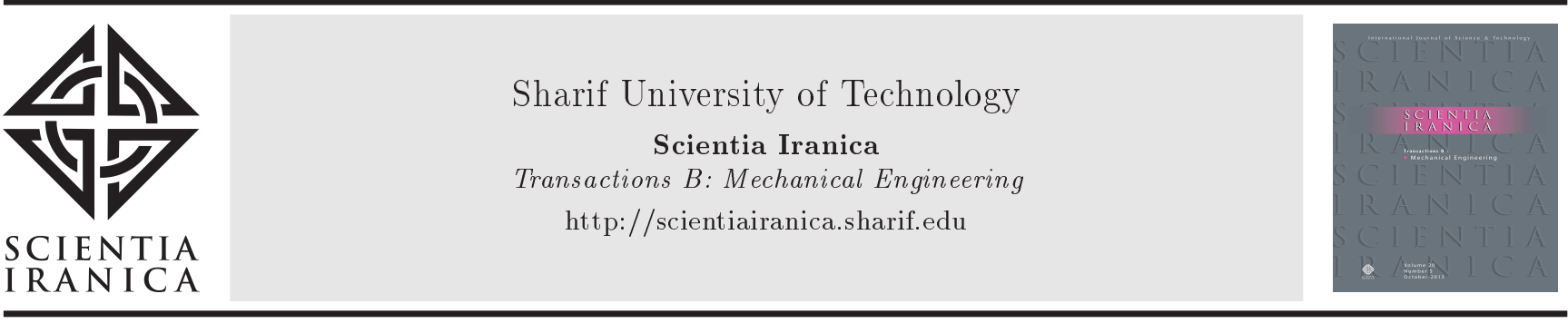

\title{
Numerical simulation of porous radiant burners under transient condition
}

\author{
H. Shabani Nejad ${ }^{a}$, S.A. Gandjalikhan Nassab ${ }^{a, *}$, and E. Jahanshahi Javaran ${ }^{b}$ \\ a. Department of Mechanical Engineering, Shahid Bahonar University of Kerman, Kerman, Iran. \\ b. Department of Energy, Institute of Science, High Technology and Environmental Sciences, Graduate University of Advanced \\ Technology, Kerman, Iran.
}

Received 15 October 2015; received in revised form 12 December 2016; accepted 18 February 2017
KEYWORDS
Porous radiant
burner;
Transient condition;
Discrete ordinate
method.

\begin{abstract}
The purpose of this study is to analyze two-dimensional rectangular porous radiant burners in order to investigate the thermal characteristics of this type of burners in starting time period. Since the solid and gas phases are not in thermal equilibrium, two separate energy equations for these two phases are solved numerically with alternative direction implicit scheme. The gas is considered non-radiative medium, and for computation of radiative heat flux through the solid phase, the Radiative Transfer Equation (RTE) is employed and solved using the Discrete Ordinates Method (DOM). It is obtained that, due to the dominant radiation effects, the required time to reach the steady gas temperature is very low. Furthermore, the effects of optical thickness and scattering albedo on the performance of Porous Radiant Burner (PRB) are investigated.
\end{abstract}

(C) 2018 Sharif University of Technology. All rights reserved.

\section{Introduction}

Nowadays, using devices that utilize more efficient combustion technologies is of particular interest to industries. Using porous materials in the structure of burners makes air-fuel mixing better and eliminates hot spots, leading to more efficient combustion with lower pollutants [1]. Combustion in porous media has further advantages compared to conventional combustion devices, such as high radiant output, high flame speed, high power density, and high combustion efficiency.

Porous Radiant Burners (PRBs) through conduction and radiation heat transfer in solid phase

\footnotetext{
*. Corresponding author. Tel.: +9832114041-7

Fax: +98 32114049;

E-mail addresses: hshabani.nejad@gmail.com (H. Shabani

Nejad); ganj110@uk.ac.ir (S.A. Gandjalikhan

Nassab)e.jahanshahi@kgut.ac.ir (E. Jahanshahi Javaran)
}

doi: $10.24200 /$ sci. 2017.4251 can recirculate heat from combustion zone toward the entrance section where air-fuel mixture enters the burner. In the flame upstream, solid-to-gas convection heat transfer preheats the reactants which results in increasing flame speeds and higher local combustion temperatures without getting high temperature at the burner exit, which reduces NOx formation.

In the last decades, many numerical studies and experimental investigations have been done on the combustion of porous media [1-6]. In order to optimize the combustion process, Brenner et al. [7] studied a 2-D pseudo homogeneous porous burner in 2000. They did not consider any radiation model, such that the radiative part has been affected by the experimental value of the effective thermal conductivity.

Talukdar et al. [8] examined a two-dimensional porous radiant burner with the detailed radiation model. Both transient and steady state characteristics were studied; however, in the transient condition, only the results of increasing time on the convective flux, 
radiative flux, gas conductive flux, and solid conductive flux were presented. They did not show the temperature distributions of gas and solid phases at different time steps. In addition, the transient behavior from the beginning of the system operation up to the steady state was not represented. Lari and Gandjalikhan Nassab [9] analyzed a one-dimensional porous burner in the transient state. In their work, the coupled energy equations for the gas and the porous medium and also the radiative transfer equation based on the two-flux method were solved numerically. Moreover, the effects of some parameters on the performance of porous radiant burner were investigated. Farzaneh et al. [10] simulated a two-dimensional porous burner in a steady condition. They solved numerically the Navier-Stokes, energy, and chemical species transport equations. The sensitivity of flame front and heat transfer characteristics to some factors was assessed. They concluded that any perturbation in heat transfer properties influences the temperature difference between the gas and solid phases and, consequently, the volumetric heat transfer between two phases.

Keshtkar and Gandjalikhan Nassab [11] studied a two-dimensional rectangular porous radiant burner in a steady condition. The gas phase was considered transparent to radiation, but the porous medium was considered to absorb, emit, and isotropically scatter radiation. They conclude that layers with large optical thickness and lower scattering albedo have high values of radiant output. In a study done by the second and third authors [12], the fluid flow in a 2-D porous heat recovery system was simulated using lattice Boltzmann method, and thermal analysis was performed by solving the governing equations of solid and gas phases. In this study, momentum and heat transfer equations were solved separately, and no temperature variation was taken into account in the fluid flow simulations. In addition, no considerable effect was observed on simulations due to considering real fluid flow in contrast to plug flow.

A useful method that is used to predict the thermal behavior of the porous radiant burners is an asymptotic analysis under simplifying assumptions. This analysis provides a practical tool for qualitative prediction of porous burner characteristics. This model successfully predicts the operational features of porous burners, such as flame location and radiant efficiency. Chandra and Nakamura [13] considered one-dimensional mathematical model for combustion of solid fuel over the porous medium. A two-temperature model with two energy equations for the gas and porous medium was employed to determine the effects of the thermal non-equilibrium between two phases. They used large activation-energy asymptotic to solve the problem. They conclude that an increase of porosity of the porous medium, thermal conductivity of porous medium, and gas flow velocity increases the moving speed of the burning front. They showed that the asymptotic simulation gives good agreement with the numerical simulation.

In the previous literature, only a few studies have been focused on the transient behavior of porous radiant burners and most of them used a simple twoflux radiation model in radiation computations. In the present work, transient characteristics of a twodimensional rectangular porous burner are numerically studied. The solid and gas phases are considered to be in non-local thermal equilibrium. Three modes of heat transfer take place simultaneously in the solid phase, while in the gas phase, only conduction and convection are considered, and the gas is considered to be transparent to radiation. Plug flow assumption is also considered. The governing equations, which are two energy equations for the gas and solid phases and also the radiative energy equation for the solid phase, are solved numerically and simultaneously to determine the thermal behavior of the system. Therefore, the porous matrix is assumed to be an emitting, absorbing, and scattering medium, and a good radiation model is necessary. To this end, Discrete Ordinates Method (DOM) is used to solve the radiative transfer equation in order to obtain heat flux distribution through solid matrix. Moreover, for the purpose of validation, the computational results are compared with the available data obtained by other researchers for the sake of favorable consistency.

\section{Porous burner configuration}

The geometry of the problem under investigation is schematically shown in Figure 1. The computational domain has $2 \mathrm{~cm}$ length and $1 \mathrm{~cm}$ height. The dimension of porous medium in the direction normal to the plane of paper ( $z$-direction) is very large compared to other dimensions ( $x$ - and $y$-directions), so a twodimensional approximation can be used. Fuel-air mixture enters from the left side with uniform temperature, $T_{g 0}$, and uniform velocity, $u_{g}$. Two main zones exist in PRBs: the preheat zone and the combustion zone. Due

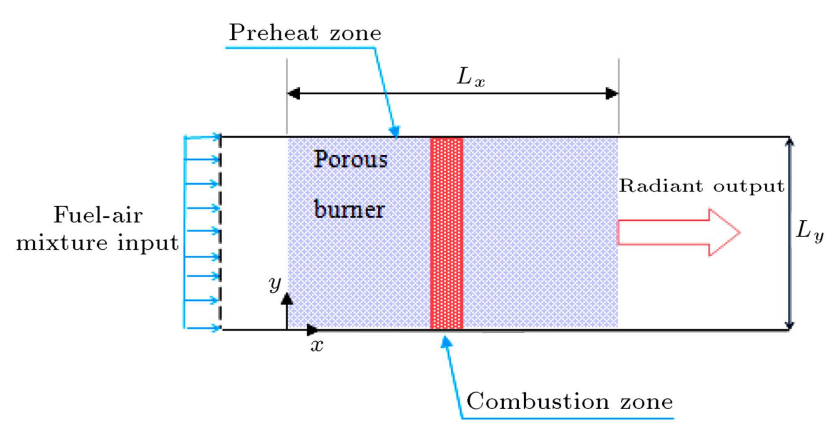

Figure 1. The schematic representation of porous radiant burner. 
to the solid conduction and radiation heat transfer from the reaction zone, the temperature of the solid phase in the preheat zone is higher than that of the gas phase. This makes enthalpy transfer by convection from solid to gas which results in preheating the gas flow, whereas, in the combustion region, enthalpy is transferred from gas to solid by reverse mechanism of the preheat zone.

\subsection{Governing equations}

Due to non-local thermal equilibrium between the solid and gas phases, separate energy equations in transient condition are considered. In addition, radiative heat transfer equation is used to find the radiative term in the solid energy equation. The following assumptions are considered in the present simulation:

- Plug flow assumption is considered;

- The solid is assumed to be a gray medium that can emit, absorb, and scatter radiation;

- Gas radiation is ignored;

- Scattering is assumed isotropic.

To save space, only the non-dimensional form of governing equations is presented here as follows:

Non-dimensional gas energy equation:

$$
\begin{aligned}
& \frac{\phi}{\Gamma \cdot P_{1}}\left(\frac{\partial^{2} \theta_{g}}{\partial \eta_{x}^{2}}+\frac{\partial^{2} \theta_{g}}{\partial \eta_{y}^{2}}\right)-\phi \frac{P_{2}}{\Gamma} \frac{\partial \theta_{g}}{\partial \eta_{x}} \\
& \quad-(1-\phi) \frac{P_{3}}{\Gamma}\left(\theta_{g}-\theta_{p}\right)+\phi \frac{P_{4}}{\Gamma} \delta(x)=\phi \frac{\partial \theta_{g}}{\partial t^{*}},
\end{aligned}
$$

Non-dimensional solid energy equation:

$$
\begin{aligned}
(1-\phi) & P_{5}\left(\frac{\partial^{2} \theta_{p}}{\partial \eta_{x}^{2}}+\frac{\partial^{2} \theta_{p}}{\partial \eta_{y}^{2}}\right)-\nabla^{*} \cdot Q_{\mathrm{rad}} \\
& +(1-\phi) P_{3}\left(\theta_{g}-\theta_{p}\right)=(1-\phi) \frac{\partial \theta_{p}}{\partial t^{*}}
\end{aligned}
$$

where $\delta(x)$ is a function defined as unity in the combustion zone and zero elsewhere. All non-dimensional parameters used in Eqs. (1) and (2) are given in the nomenclature.

The convection heat transfer between the gas and solid phases couples their equations together. Therefore, it is necessary to have an appropriate relation for the convective heat transfer coefficient. To this end, the following relationship is used [14]:

$$
h=k_{g}\left[2+1.1 \operatorname{Pr}^{1 / 3} \operatorname{Re}_{p}^{0.6}\right] / d_{p},
$$

where $\operatorname{Re}_{p}$ is the particle-based Reynolds number which is defined as: $\operatorname{Re}_{p}=\frac{\rho_{g} u_{g} d_{p}}{\mu_{g}}$.
The dimensionless divergence of radiative heat flux, $\nabla^{*} \cdot Q_{\text {rad }}$, which should be calculated from the radiative transfer equation, can be written as follows:

$$
\nabla^{*} \cdot Q_{\mathrm{rad}}=\frac{\partial Q_{x}}{\partial \eta_{x}}+\frac{\partial Q_{y}}{\partial \eta_{y}} .
$$

The non-dimensional radiative transfer equation in an emitting, absorbing, and scattering medium can be written in general form as follows [15]:

$$
\begin{gathered}
\hat{s} . \nabla^{*} I^{*}(\vec{r}, \hat{s})=-\tau_{1} I^{*}(\vec{r}, \hat{s})+\tau_{1} I_{b}^{*}(\vec{r}) \\
+\frac{\tau_{2}}{4 \pi} \int_{\omega^{\prime}=4 \pi} I^{*}\left(\vec{r}, \hat{s}^{\prime}\right) \varphi\left(\vec{r}, \hat{s}, \hat{s}^{\prime}\right) d \Omega^{\prime} .
\end{gathered}
$$

The critical issue in the solution of the radiative transfer equation for the porous burner is the calculation of the radiative properties of the medium. In this study, optical thickness and scattering albedo are considered as representatives of other properties. Optical thickness, $\tau_{0}$, which shows the ability of the media to absorb and emit thermal radiation, is defined as $\tau_{0}=\beta \times L_{x}$, where $\beta$ is the extinction coefficient. In addition, optical thickness measures the attenuation of the transmitted radiant power in a material. Another important radiant property of porous materials, which can affect the thermal behavior of PRBs, is scattering albedo, defined as the ratio of scattering coefficient to extinction coefficient $\left(\omega=\sigma_{s} / \beta\right)$. In general, extinction coefficient is a function of the type of material, construction of the material, and operating temperature. The scattering albedo is a strong function of material and relatively independent of structure and temperature [16]. It should be noted that the range of the present values considered for optical thickness and scattering albedo is according to [16].

Once the RTE with appropriate boundary conditions was solved for the computation of radiant intensities, the radiative heat flux can be calculated from:

$$
Q_{\mathrm{rad}}(\vec{r})=\int_{4 \pi} I^{*}(\vec{r}, \hat{s}) \hat{s} d \Omega .
$$

\subsection{Initial and boundary conditions}

For numerical solution of the gas and solid phases, energy equations, and also the RTE, an appropriate set of initial and boundary conditions are needed.

Solid and gas phases are assumed initially to be at ambient temperature as follows:

$$
\theta_{g}=\theta_{\infty} \quad \text { and } \quad \theta_{p}=\theta_{\infty} \quad \text { at } \quad t^{*}=0 .
$$

The following boundary conditions are applied to solve the gas energy equation: 


$$
\begin{aligned}
& \theta_{g}=\theta_{g 0} \quad \text { at } \quad \eta_{x}=0, \\
& \frac{\partial \theta_{g}}{\partial \eta_{x}}=0 \quad \text { at } \quad \eta_{x}=1, \\
& \frac{\partial \theta_{g}}{\partial \eta_{y}}=P_{6}\left(\theta_{g}-\theta_{\infty}\right) \quad \text { at } \quad \eta_{y}=0, \\
& \frac{\partial \theta_{g}}{\partial \eta_{y}}=-P_{6}\left(\theta_{g}-\theta_{\infty}\right) \quad \text { at } \quad \eta_{y}=\eta_{L_{y}} .
\end{aligned}
$$

In inlet and outlet sections of the solid phase, due to convection heat transfer between the gas and solid phases and radiation heat transfer between solid matrix and its surrounding, the following boundary conditions are used:

$$
\begin{aligned}
& \operatorname{Bi}\left(\theta_{g}-\theta_{p}\right)+\frac{\varepsilon_{p_{1}}}{P_{5}}\left(\theta_{i}^{4}-\theta_{p}^{4}\right)=-\frac{\partial \theta_{p}}{\partial \eta_{x}} \\
& \text { at } \eta_{x}=0, \\
& \operatorname{Bi}\left(\theta_{p}-\theta_{g}\right)+\frac{\varepsilon_{p_{2}}}{P_{5}}\left(\theta_{p}^{4}-\theta_{e}^{4}\right)=-\frac{\partial \theta_{p}}{\partial \eta_{x}}
\end{aligned}
$$$$
\text { at } \eta_{x}=1 \text {. }
$$

Boundary conditions at upper and lower surfaces of the solid matrix are as follows:

$$
\begin{aligned}
& \frac{\partial \theta_{p}}{\partial \eta_{y}}=P_{7}\left(\theta_{p}-\theta_{\infty}\right) \quad \text { at } \quad \eta_{y}=0, \\
& \frac{\partial \theta_{p}}{\partial \eta_{y}}=-P_{7}\left(\theta_{p}-\theta_{\infty}\right) \quad \text { at } \quad \eta_{y}=\eta_{L Y} .
\end{aligned}
$$

In order to solve the RTE, suitable boundary conditions should be considered. It is assumed that radiant intensities, $B_{1}$ and $B_{2}$, enter the domain from inlet and outlet sections. Therefore, the following boundary conditions are applied to the radiative transfer equation:

$$
\begin{aligned}
& I^{* m}\left(0, \eta_{y}\right)=\frac{B_{1}^{\prime}}{\pi} \quad \text { at } \quad \eta_{x}=0 \\
& I^{* m}\left(0, \eta_{y}\right)=\frac{B_{2}^{\prime}}{\pi} \quad \text { at } \quad \eta_{x}=1 \\
& I^{* m}\left(\eta_{x}, 0\right)=\varepsilon_{B} \frac{\sigma \theta_{B}^{4}}{\pi} \\
& +\frac{\rho_{B}}{\pi} \int_{\hat{n} . \hat{s}^{\prime}<0}\left|\hat{n} . \hat{s}^{\prime}\right| I^{*}\left(\eta_{x}, 0, \hat{s}^{\prime}\right) d \Omega^{\prime} \text { at } \eta_{y}=0 \\
& I^{* m}\left(\eta_{x}, \eta_{L_{y}}\right)=\varepsilon_{T} \frac{\sigma \theta_{T}^{4}}{\pi} \\
& +\frac{\rho_{T}}{\pi} \int_{\hat{n} . \hat{s}^{\prime}<0}\left|\hat{n} . \hat{s}^{\prime}\right| I^{*}\left(\eta_{x}, \eta_{L_{y}}, \hat{s}^{\prime}\right) d \Omega^{\prime} \\
& \text { at } \eta_{y}=\eta_{L_{y}} .
\end{aligned}
$$

\subsection{Discrete ordinates method $\left(S_{N}\right.$ approximation)}

Due to the complex nature of RTE, many methods have been proposed to simplify this equation. One of the commonly used methods to solve the radiative transfer equation is the discrete ordinates method. This method is based on the discrete representation of the directional behavior of the radiative intensity. DOM transforms the equation of heat transfer into a set of simultaneous partial differential equations. The name $S_{N}$ approximation shows that $N$ different direction cosines are used for each principal direction. Altogether, there are $n=N(N+2)$ different directions to be considered in three-dimensional computational domain. The general form of RTE is solved for a set of $n$ different directions $\hat{s}_{i}, i=1,2, \ldots, n$ [14], such that the integrals over direction are replaced by the following relation:

$$
\int_{4 \pi} f(\hat{s}) d \Omega \approx \sum_{i=1}^{n} w_{i} f\left(\hat{s}_{i}\right),
$$

where $w_{i}$ is the quadrature weights associated with directions $\hat{s}_{i}$.

Hence, the RTE is approximated by a set of $n$ differential equations as follows:

$$
\begin{aligned}
& \hat{S} . \nabla^{*} I^{*}\left(\vec{r}, \hat{s}_{i}\right)=-\tau_{0} I^{*}\left(\vec{r}, \hat{s}_{i}\right)+\tau_{1} I_{b}^{*}(\vec{r}) \\
& +\frac{\tau_{2}}{4 \pi} \sum_{j=1}^{n} w_{j} I^{*}\left(\vec{r}, \hat{s}_{j}\right) \varphi^{*}\left(\vec{r}, \hat{s}_{i}, \hat{s}_{j}\right) \\
& i=1,2, \ldots, n .
\end{aligned}
$$

For 2-D Cartesian coordinate problems, Eq. (21) becomes:

$$
\begin{aligned}
\xi^{m} \frac{\partial I^{m}}{\partial x} & +\eta^{m} \frac{\partial I^{m}}{\partial y}=-\beta I^{m}+\sigma_{a} I_{b} \\
& +\frac{\sigma_{s}}{4 \pi} \sum_{m^{\prime}} w^{m^{\prime}} \varphi^{m^{\prime} m} I^{m^{\prime}}
\end{aligned}
$$

where $m$ and $m^{\prime}$ are outgoing and incoming directions, respectively, and $\xi^{m}$ are $\eta^{m}$ the components of unit vector $\hat{s}_{i}$. Finally, the radiative heat flux inside the porous medium can be determined by the following equation:

$$
Q_{\mathrm{rad}}(\vec{r})=\int_{4 \pi} I^{*}\left(\vec{r}, \hat{s}_{i}\right) \hat{s}_{i} d \Omega \approx \sum_{i=1}^{n} w_{i} I^{*}(\vec{r}) \hat{s}_{i} .
$$

Numerically, using the finite difference method, the radiative intensity at each node in desired directions can be determined from:

$$
I_{i, j}^{m}=\frac{I X^{m}+I Y^{m}+\sigma_{a} I_{b, i, j}+S^{m}}{\beta+X^{m} \operatorname{sign}\left(X^{m}\right)+Y^{m} \operatorname{sign}\left(Y^{m}\right)},
$$


where:

$$
\begin{aligned}
& X^{m}=\frac{\xi^{m}}{\Delta x}, \quad Y^{m}=\frac{\eta^{m}}{\Delta y}, \\
& S^{m}=\frac{\sigma_{s}}{4 \pi} \sum_{m^{\prime}} w^{m^{\prime}} \phi^{m^{\prime} m} I_{i, j}^{m^{\prime}}, \\
& I X^{m}=X^{m} u_{0}\left(X^{m}\right) I^{m}{ }_{i-1, j}-X^{m} u_{0}\left(-X^{m}\right) I_{i+1, j}^{m}, \\
& I Y^{m}=Y^{m} u_{0}\left(Y^{m}\right) I^{m}{ }_{i, j-1}-Y^{m} u_{0}\left(-Y^{m}\right) I^{m}{ }_{i, j+1}, \\
& \operatorname{sign}(x)= \begin{cases}1 & x>0 \\
-1 & x<0\end{cases} \\
& u_{0}(x)= \begin{cases}1 & x>0 \\
0 & x<0\end{cases}
\end{aligned}
$$

Therefore, from Eq. (24), the value of radiant intensities at each nodal point $(i, j)$ can be computed.

\subsection{Method of solution}

In order to obtain the temperature distribution of the gas and solid phases and also the values of radiative heat fluxes in the solid phase, the governing equations with related boundary conditions are solved iteratively. Because of transient behavior of the energy equations, the Alternative Direction Implicit (ADI) scheme is used to discretize these equations. In numerical analysis, the ADI scheme is an implicit finite difference method for solving parabolic, hyperbolic, and elliptic partial differential equations. The advantage of the ADI is that the equations, which have to be solved in each step, have a simpler structure and can be solved efficiently with the tri-diagonal matrix algorithm. The idea behind the ADI is to split the finite difference equations into two ones: one with the $x$-derivative taken implicitly and other with the $y$-derivative.

With the help of the above numerical methods, the temperature distributions in the solid and gas phases and also the values of radiant heat flux at each nodal point and time step can be computed by the following procedure:

Step 1. The initial values of temperature and radiative heat flux are considered;

Step 2. The tri-diagonal matrix algorithm is applied to solve the discrete form of the gas energy equation to obtain the gas temperature distribution;

Step 3. The radiative transfer equation is solved to determine the values of $I^{*}, Q_{\mathrm{rad}}$, and $\nabla^{*}, Q_{\mathrm{rad}}$ at each nodal point;

Step 4. The porous energy equation (Eq. (2)), with the values of $\theta_{g}$ and $\nabla^{*} . Q_{\text {rad }}$ obtained from Steps 2 and 3, is used to compute the temperature of solid phase at each node;
Step 5. Steps 2 to 4 are repeated until convergence is obtained.

It should be noted that convergence is achieved when changes in temperature and radiative intensity between two consecutive iterations do not exceed $10^{-3}$ at each grid point. Steady-state condition is achieved when temperature changes in two consecutive time steps are less than $10^{-5}$.

\section{Comparison of the numerical results in the steady condition with those in the literature}

In order to examine the validity of the present numerical code, the numerical results for a test case in steady condition are compared with those obtained by Talukdar et al. [8]. They analyzed a 2D rectangular porous radiant burner. In their work, the solid phase was assumed absorbing, emitting, and scattering, while the gas phase was considered transparent to radiation. They used the collapsed dimension method to solve the radiative term of solid energy equation. The nondimensional parameters of the PRB are based on the values from Talukdar et al. [8] given in Table 1. The steady-state gas temperature distribution is shown in Figure 2. Radiation and conduction heat transfer of

Table 1. Non-dimensional parameters.

\begin{tabular}{cc}
\hline Parameters & Values from Talukdar et al. [8] \\
\hline$P_{1}$ & 1.2 \\
$P_{2}$ & 33.33 \\
$P_{3}$ & 333.33 \\
$P_{4}$ & $10^{8}$ \\
$P_{5}$ & 66.66 \\
$\tau_{0}$ & 1.0 \\
$\omega$ & 0.0 \\
$\Gamma$ & $3.33 \times 10^{-3}$ \\
$\mathrm{Bi}$ & 5 \\
\hline
\end{tabular}

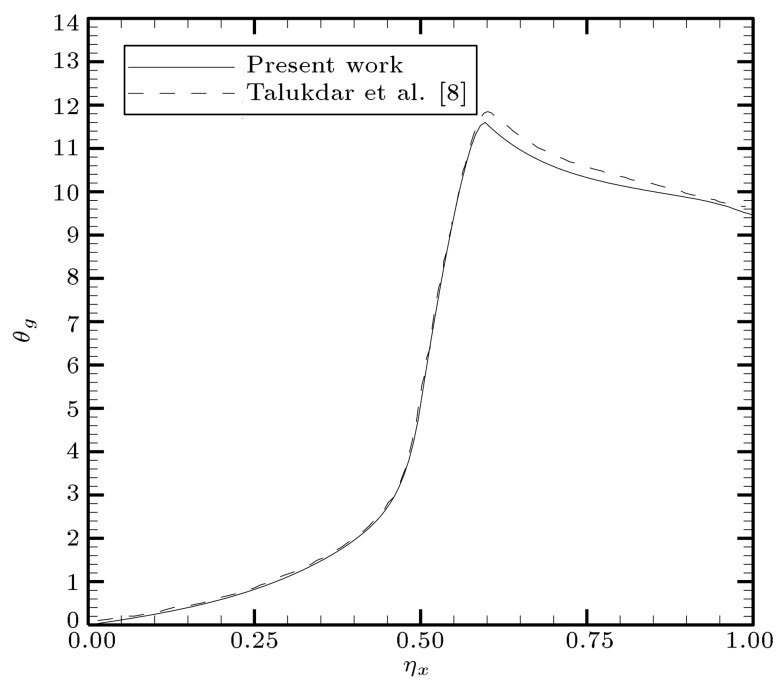

Figure 2. Gas temperature distribution along PRB. 
the solid phase recirculates heat from the combustion zone to the entrance zone of the porous burner; consequently, convection heat transfer with porous matrix preheats the fuel-air mixture. Due to the heat released in the combustion region, the maximum temperature of the gas phase occurs in this zone. Downstream this zone, the gas temperature decreases by converting the gas enthalpy to thermal radiation towards the outlet section. The increase in the gas temperature up to the heat generation zone is clearly seen in this figure after which the temperature decreases towards the outlet section. However, satisfactory agreement is found between the present results and those of [8]. Small discrepancy seen in downstream the combustion zone of the porous burner may be due to different boundary conditions used in the existing section in these studies, deteriorating the numerical results in the upstream of this section.

\section{Results and discussion}

The main purpose of this study is to analyze the transient behavior of a porous radiant burner. Optical thickness, $\tau_{0}$, is the potential of a medium in radiation heat transfer. The greater the optical thickness, the better the performance of the medium in radiation transfer. To illustrate this effect, time history of the burner for two optical thicknesses is shown in Figures 3 and 4 . In both figures, by increasing time, the gas temperatures increase until reaching a steady-state condition. The heat generation zone is situated in the middle of porous layer, so the maximum values of temperatures occur in the combustion zone due to

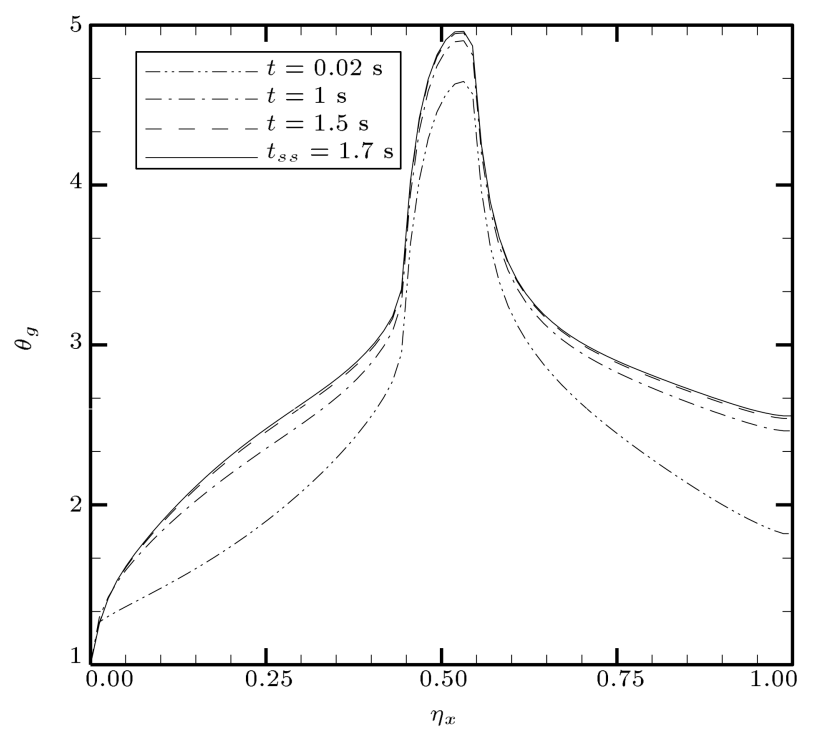

Figure 3. The time history of gas temperature at centerline with $\tau_{0}=1.0\left(P_{1}=0.24, P_{2}=405.2\right.$, $P_{3}=18.66 \times 10^{4}, P_{4}=44.7 \times 10^{3}, P_{5}=333.22, P_{6}=16.0$, $\left.P_{7}=0.2, \mathrm{Bi}=8.0, \omega=0.0, \Gamma=3.47 \times 10^{-3}\right)$.

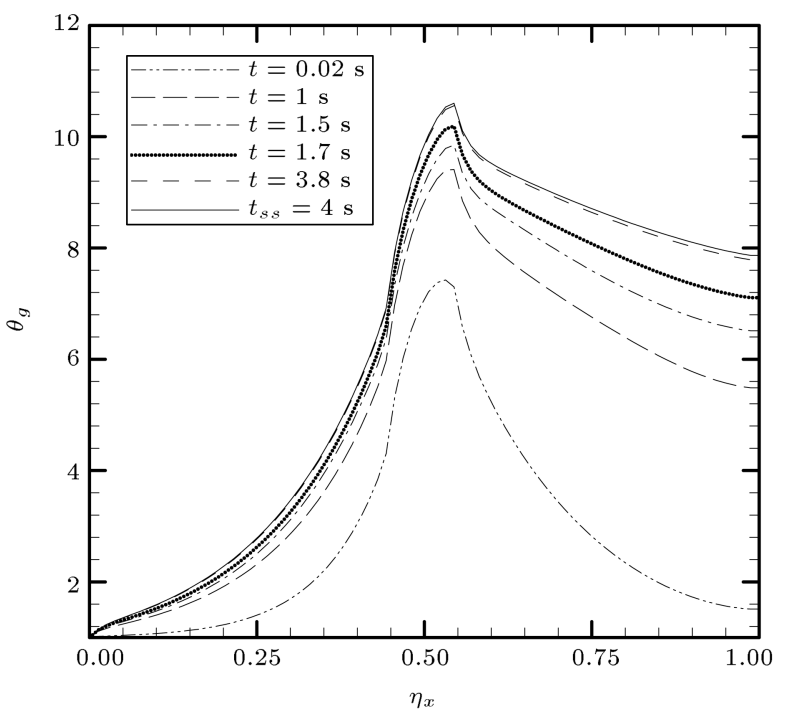

Figure 4. The time history of gas temperature at center line with $\tau_{0}=0.001\left(P_{1}=0.24, P_{2}=405.2\right.$,

$P_{3}=18.66 \times 10^{4}, P_{4}=44.7 \times 10^{3}, P_{5}=333.22, P_{6}=16.0$, $\left.P_{7}=0.2, \mathrm{Bi}=8.0, \omega=0.0, \Gamma=3.47 \times 10^{-3}\right)$.

heat releasing phenomenon. In addition, it is seen in these figures that the gas phase is preheated by increasing time; this is due to the convection heat transfer with solid phase. The required time to reach a steady-state condition in case of $\tau_{0}=1$, due to the dominance of radiation effects, is less than that of $\tau_{0}=0.001$. It means that radiation phenomenon caused the temperature field to become steady in a short time period. Besides, one can find from Figures 3 and 4 that the temperature distributions have similar patterns at each time step, and they reach steady condition during a very short time period.

Gas and solid temperature distributions in the steady condition are presented in Figure 5. In the premixed region, the solid temperature due to the radiative fluxes from combustion zone is higher than the gas temperature. Due to the heat generation in the combustion zone, the gas temperature increases sharply before this domain, and the porous temperature has a similar trend due to a high rate of convection heat transfer between these two phases. In addition, it is depicted from Figure 5 that the maximum values of gas and solid temperatures occur inside the combustion zone, where there is a heat source domain inside the gas flow.

Figure 6 shows the radiative heat flux distribution of the solid phase along the flow direction. The value of downstream radiative flux, $Q^{+}$, at the end of solid matrix, $\eta_{x}=1$, is called radiant output, which is a very important parameter in increasing radiant burner efficiency. The upstream radiative heat flux, $Q^{-}$, at the inlet of porous burner, $\eta_{x}=0$, is the energy loss which decreases the burner efficiency. It is desirable that this value should be very low to have more efficient PRB. 


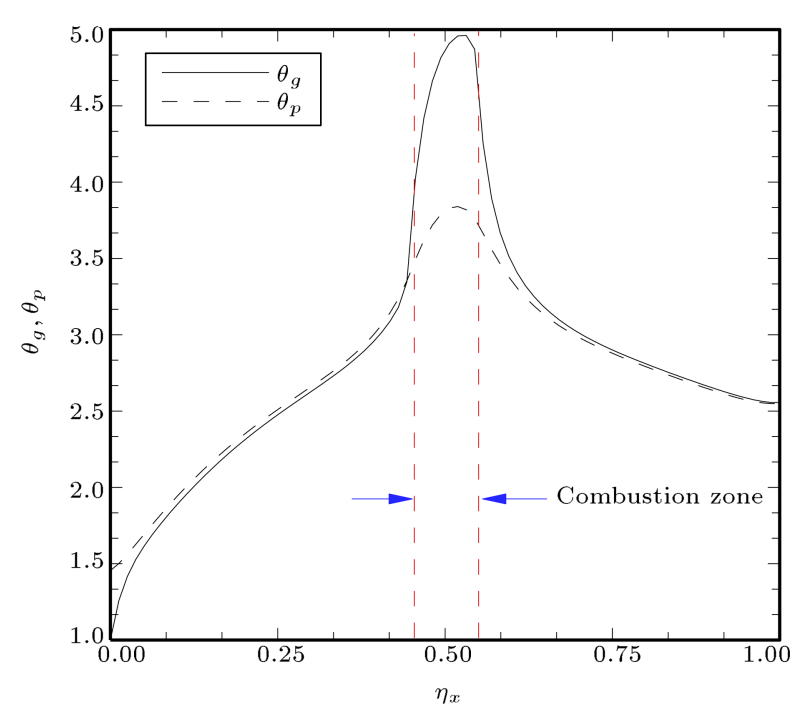

Figure 5. Gas and solid temperature distributions in steady condition at $\eta_{y}=0.5\left(P_{1}=0.24, P_{2}=405.2\right.$, $P_{3}=18.66 \times 10^{4}, P_{4}=44.7 \times 10^{3}, P_{5}=333.22, P_{6}=16.0$, $\left.P_{7}=0.2, \mathrm{Bi}=8.0, \tau_{0}=1.0, \omega=0.0, \Gamma=3.47 \times 10^{-3}\right)$.

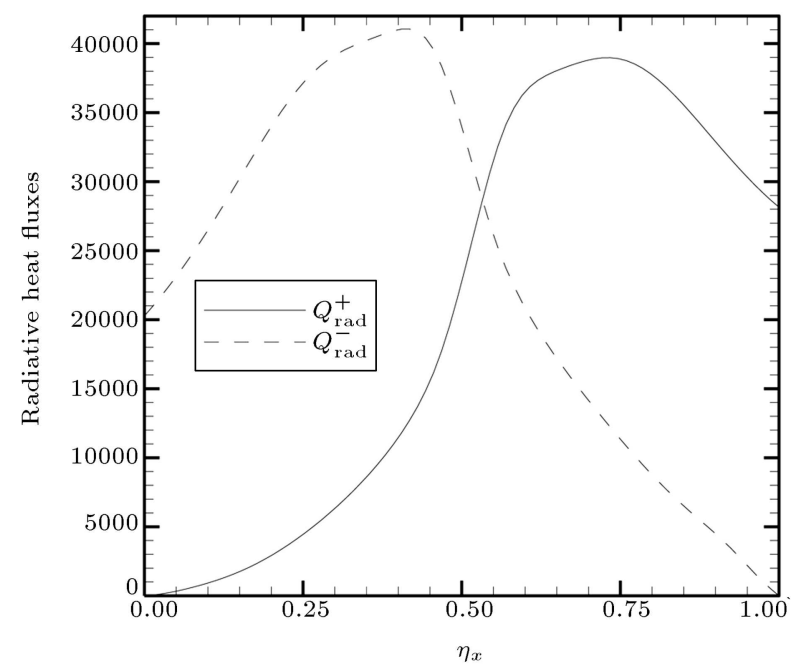

Figure 6. Radiative flux distribution at steady-state condition in centerline $\left(P_{1}=0.24, P_{2}=405.2\right.$, $P_{3}=18.66 \times 10^{4}, P_{4}=44.7 \times 10^{3}, P_{5}=333.22, P_{6}=16.0$, $P_{7}=0.2, \mathrm{Bi}=8.0, \tau_{0}=1.0, \omega=0.0, \Gamma=3.47 \times 10^{-3}$, $\left.B_{1}=0.0, B_{2}=0.0\right)$.

The effect of optical thickness on thermal characteristics of PRBs is studied in Figure 7, in which the gas and solid temperature distributions along the centerline in the steady condition are plotted. These figures show that the temperature distributions are much affected by the optical thickness so that both gas and porous temperatures decrease with an increase in $\tau_{0}$. This behavior can be explained by noticing this fact that the porous burner with high optical thickness can convert more gas enthalpy to thermal radiation, which causes a decrease in maximum gas temperature and an increase in radiant output. As depicted in Figure 7, the high val-

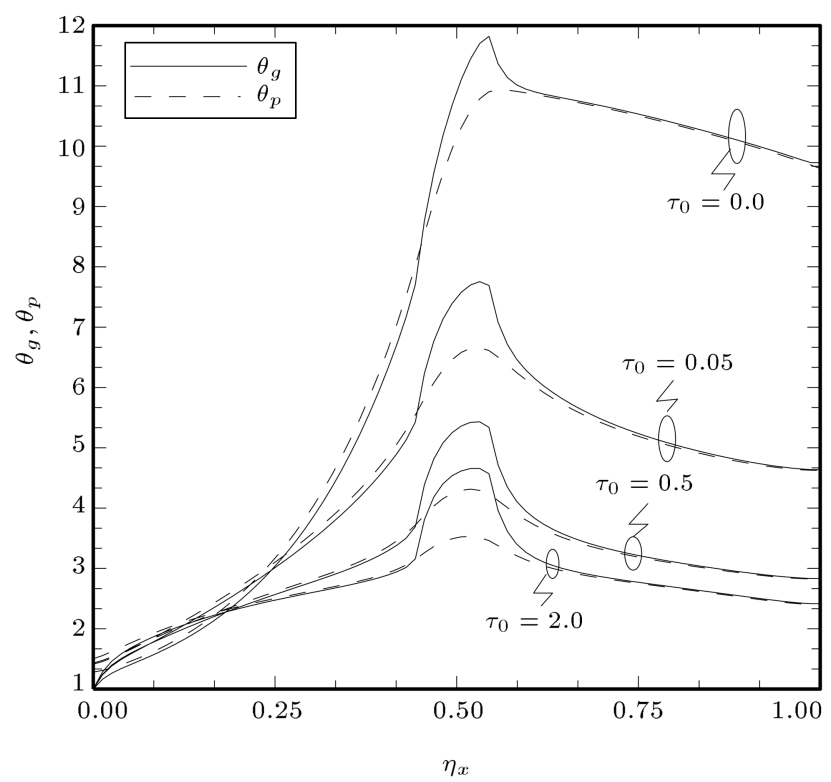

Figure 7. Effect of optical thickness on the temperatures of the gas and solid phases $\left(P_{1}=0.24, P_{2}=405.2\right.$,

$P_{3}=18.66 \times 10^{4}, P_{4}=44.7 \times 10^{3}, P_{5}=333.22, P_{6}=16.0$, $\left.P_{7}=0.2, \mathrm{Bi}=8.0, \omega=0.0, \Gamma=3.47 \times 10^{-3}\right)$.

ues of gas and porous temperatures take place in porous burners with zero optical thickness in which there is not any energy conversion from gas enthalpy into thermal radiation, so that all of the thermal energy released from combustion process leads to high temperature gas flow through the burner. Accordingly, large variations of temperature with changing optical thickness from 0 to 2 are observed in Figure 7 . In addition, in the preheat zone where there is no heat generation, the effect of optical thickness on the temperature is less in comparison to the combustion zone.

The gas and solid temperatures for different scattering albedos are represented in Figure 8. This figure shows that decreasing the scattering albedo reduces the maximum temperatures of the gas and solid phases due to a decrease in the conversion of enthalpy to thermal radiation.

Porosity or void fraction, a measure of the void spaces in the porous material, is defined as the volume of voids over the total volume. To show the effect of this parameter, gas temperature distributions for three porosities are shown in Figure 9. As seen in this figure, by increasing porosity which leads to a decrease in the volume of solid phase, the rate of energy conversion between gas enthalpy and thermal radiation decreases, and finally, high temperature gas flows through the burner without considerable temperature changes.

Furthermore, the performance of PRBs is usually defined as radiant efficiency, which is the ratio of the radiant output to the total amount of energy released via the combustion process as follows [17]: 


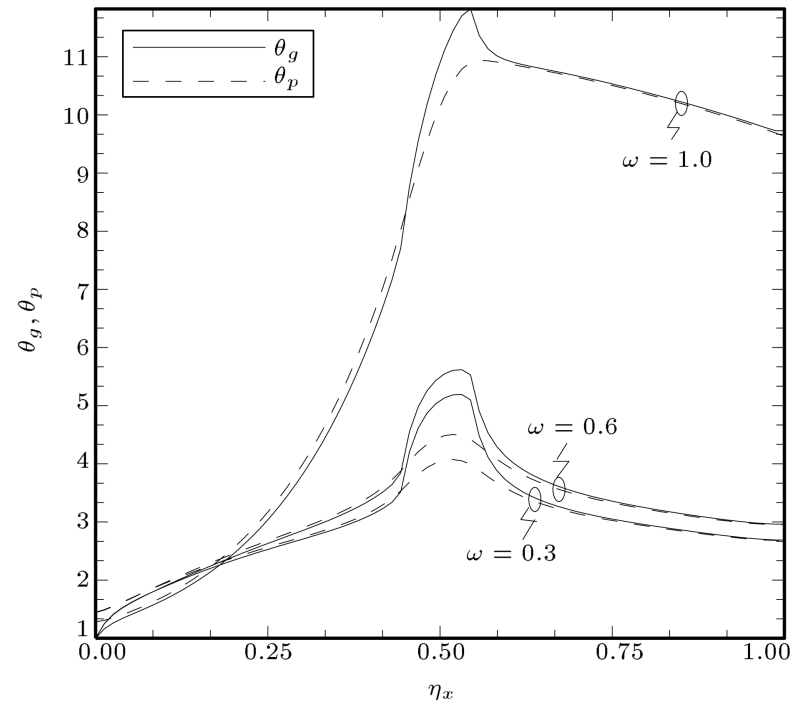

Figure 8. The effect of scattering albedo on the temperatures of the gas and solid phases $\left(P_{1}=0.24\right.$, $P_{2}=405.2, P_{3}=18.66 \times 10^{4}, P_{4}=44.7 \times 10^{3}$, $P_{5}=333.22, P_{6}=16.0, P_{7}=0.2, \mathrm{Bi}=8.0, \tau_{0}=1.0$, $\left.\Gamma=3.47 \times 10^{-3}\right)$.

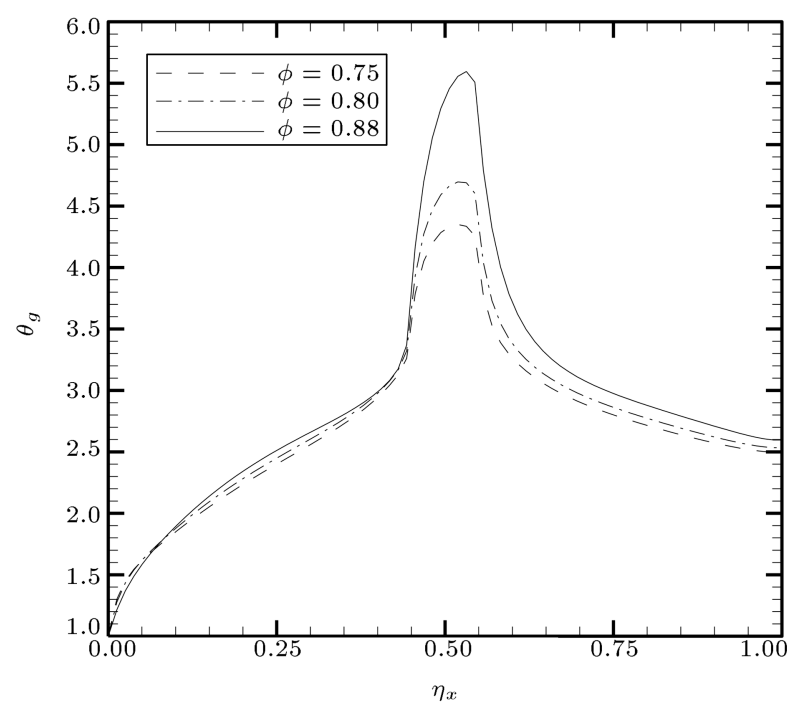

Figure 9. The effect of porosity on the gas temperature $\left(P_{1}=0.24, P_{2}=405.2, P_{3}=18.66 \times 10^{4}, P_{4}=44.7 \times 10^{3}\right.$, $P_{5}=333.22, P_{6}=16.0, P_{7}=0.2, \mathrm{Bi}=8.0, \tau_{0}=1.0$, $\omega=0.0, \Gamma=3.47 \times 10^{-3}$ ).

$$
\eta_{r}=\frac{\text { Heat released by radiation at output }}{\text { Heat released by complete combustion }} \times 100 \text {. }
$$

In Figure 10, the time history of the variation of radiant efficiency is presented. This figure shows zero value for the burner efficiency at the starting time; therefore, it increases rapidly with time and reaches its steady-state value. Figure 11 shows the effect of the optical thickness on the radiant efficiency. Also, in Figure 12, the effect of scattering albedo on the radiant efficiency is seen. As observed in Figure 11, the higher

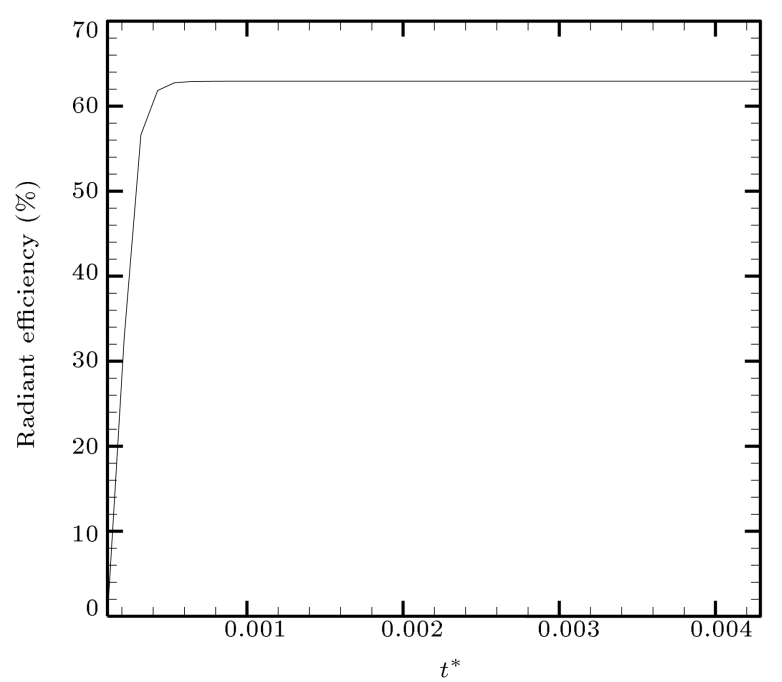

Figure 10. The time history of burner efficiency $\left(P_{1}=0.24, P_{2}=405.2, P_{3}=18.66 \times 10^{4}, P_{4}=44.7 \times 10^{3}\right.$, $P_{5}=333.22, P_{6}=16.0, P_{7}=0.2, \mathrm{Bi}=8.0, \tau_{0}=1.0$, $\omega=0.0, \Gamma=3.47 \times 10^{-3}$ ).

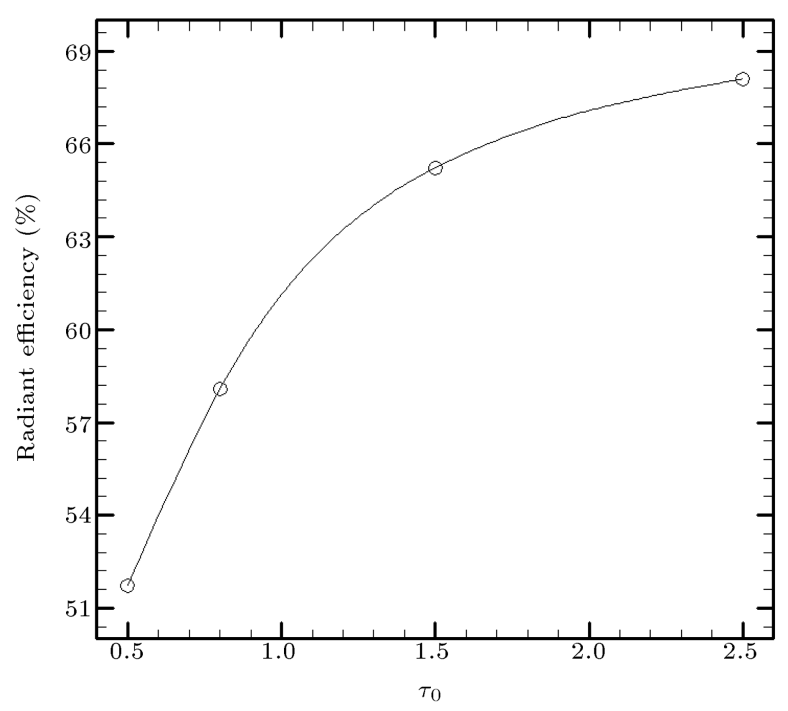

Figure 11. The effect of optical thickness on the radiant efficiency $\left(P_{1}=0.24, P_{2}=405.2, P_{3}=18.66 \times 10^{4}\right.$, $P_{4}=44.7 \times 10^{3}, P_{5}=333.22, P_{6}=16.0, P_{7}=0.2, \mathrm{Bi}=$ $\left.8.0, \omega=0.0, \Gamma=3.47 \times 10^{-3}\right)$.

the optical thickness, the more the radiant efficiency. In addition, reverse phenomenon happens in case of the scattering albedo. It can be concluded that using porous layers with high optical thickness and small scattering albedo in the construction of radiant burners leads to an increase in the performance of PRBs by converting more thermal energy from gas enthalpy to thermal radiation.

\section{Conclusions}

The thermal characteristics of a porous radiant burner in the transient condition was studied numerically in 


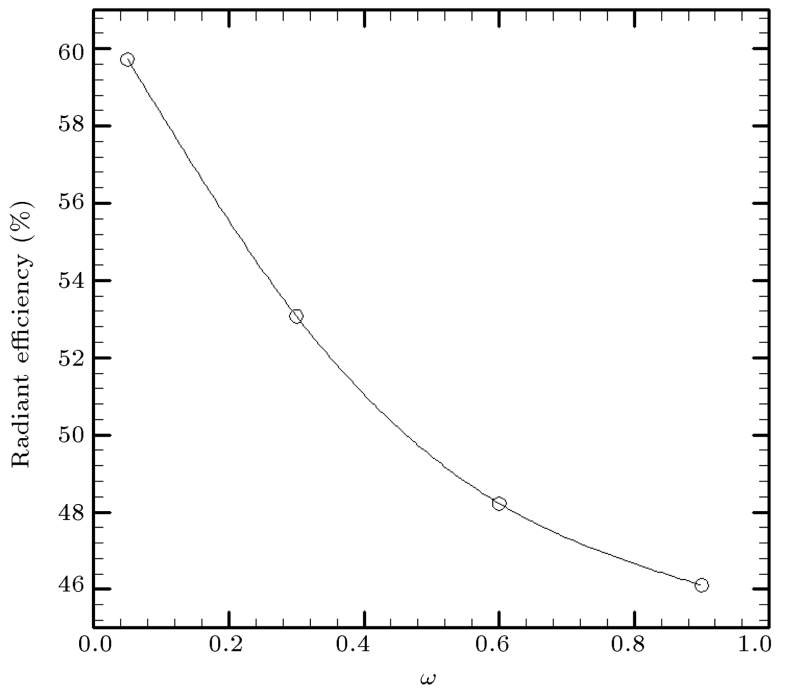

Figure 12. The effect of scattering albedo on the radiant efficiency $\left(P_{1}=0.24, P_{2}=405.2, P_{3}=18.66 \times 10^{4}\right.$, $P_{4}=44.7 \times 10^{3}, P_{5}=333.22, P_{6}=16.0, P_{7}=0.2, \mathrm{Bi}=$ 8.0, $\left.\tau_{0}=1.0, \Gamma=3.47 \times 10^{-3}\right)$.

this work. To this end, a 2D solid matrix in Cartesian coordinates with combustion chamber located in its middle was considered. The governing equations include the gas and solid energy equations, and the radiative transfer equation is solved by numerical method. The alternative direction implicit scheme and discrete ordinates method are used to discretize the energy equations and solve the RTE, respectively. Numerical results show that the required time to reach a steadystate condition is relatively short in porous radiant burners. This phenomenon occurs due to the nature of radiation mode whose most effective contribution in the transferring energy is instantaneous, so that the transient time period is only as a result of the existence of conduction and convection heat transfer modes. It can be concluded that, in the analysis of such thermal systems, the duration of transient thermal behavior is not important; therefore, the investigation can be considered in a steady-state condition. In addition, findings show that two important radiative properties on the behavior of PRBs are the optical thickness and scattering albedo. To construct radiant burners, it is better to use porous media with large optical thickness and small scattering albedo leading to PRBs with high performance.

\section{Nomenclature}

$A \quad$ Surface area per unit volume $\left(\mathrm{m}^{2} / \mathrm{m}^{3}\right)$

$B_{1,2} \quad$ Incoming radiations $\left(\mathrm{W} / \mathrm{m}^{2}\right)$

$B_{1,2}^{\prime} \quad$ Non-dimensional incoming radiations, $B_{1,2} / \sigma T_{g 0}^{4}$

Bi Biot number, $h L_{x} / k_{p}$

\section{Greek symbols}

Special heat of gas $(\mathrm{J} / \mathrm{kgK})$

Special heat of solid $(\mathrm{J} / \mathrm{kgK})$

Particle diameter $(\mathrm{m})$

Convective heat transfer coefficient $\left(\mathrm{W} / \mathrm{m}^{2} K\right)$ Intensity $\left(\mathrm{W} / \mathrm{m}^{2}\right)$

Non-dimensional intensity, $I / \sigma T_{g 0}^{4}$

Gas thermal conductivity coefficient $(\mathrm{W} / \mathrm{mK})$

Solid thermal conductivity coefficient $(\mathrm{W} / \mathrm{mK})$

Length of porous medium (m)

Height of porous medium (m)

Nusselt number, $h L_{x} / k_{g}$

The ratio of solid radiation to gas conduction, $\sigma T_{g 0}{ }^{3} L_{x} / k_{g}$

The ratio of gas convection to solid radiation, $\rho_{g} c_{g} u_{g} / \sigma T_{g 0}^{3}$

\section{The ratio of convection between two} phases to solid radiation, $h A L_{x} / \sigma T_{g 0}^{3}$

The ratio of combustion heat to solid radiation, $L_{x} \dot{Q} / \sigma T_{g 0}^{4}$

The ratio of solid conduction to solid radiation, $k_{p} / \sigma T_{g 0}^{3} L_{x}$

The ratio of gas convection to gas conduction at boundaries, $h_{w g} L_{x} / k_{p}$

The ratio of solid convection to solid conduction at $h_{w p} L_{x} / k_{p}$

Radiative heat flux $\left(W / m^{2}\right)$

Non-dimensional radiative heat flux, $q_{\mathrm{rad}} / \sigma T_{g 0}^{4}$

Particle-based Reynolds number, $\rho_{g} u_{g} d_{p} / \mu_{g}$

Direction vector in RTE

Temperature (K)

Initial temperature of gas $(\mathrm{K})$

Ambient temperature (K)

Time (s)

Non-dimensional time, $\sigma T_{g 0}^{3} t / \rho_{p} c_{p} L_{x}$ Gas velocity along $x$ direction $(\mathrm{m} / \mathrm{s})$

Coordinate along flow direction $(\mathrm{m})$

Coordinate perpendicular to the flow direction $(\mathrm{m})$

\footnotetext{
Extinction coefficient $\left(\mathrm{m}^{-1}\right), \sigma_{a}+\sigma_{s}$
} 
$\nabla^{*} \quad$ Non-dimensional gradient operator, $L_{x} \nabla$

$\Delta \eta_{x} \quad$ Non-dimensional grid spacing along $x$-axis

$\Delta \eta_{y} \quad$ Non-dimensional grid spacing along $y$-axis

$\varepsilon \quad$ Emissivity

$\eta_{x} \quad$ Non-dimensional $x$ coordinate, $x / L_{x}$

$\eta_{y} \quad$ Non-dimensional $y$ coordinate, $y / L_{X}$

$\Gamma \quad$ Non-dimensional parameter, $\rho_{g} c_{g} / \rho_{p} c_{p}$

$\theta \quad$ Non-dimensional temperature, $T / T_{g 0}$

$\rho_{g} \quad$ Gas density $\left(\mathrm{m}^{3} / \mathrm{kg}\right)$

$\rho_{p} \quad$ Solid density $\left(\mathrm{m}^{3} / \mathrm{kg}\right)$

$\rho_{w} \quad$ Wall reflection coefficient

$\sigma \quad$ Stephan-Boltzmann coefficient $\left(\mathrm{w} / \mathrm{m}^{2} K^{4}\right)$

$\sigma_{a} \quad$ Absorption coefficient $\left(\mathrm{m}^{-1}\right)$

$\sigma_{s} \quad$ Scattering coefficient $\left(\mathrm{m}^{-1}\right)$

$\theta_{g, p} \quad$ Non-dimensional temperature,

$T_{g, p} / T_{g 0}$

$\tau_{0} \quad$ Optical thickness, $\beta L_{x}$

$\tau_{1} \quad$ Non-dimensional parameter, $\sigma_{a} L_{x}$

$\tau_{2} \quad$ Non-dimensional parameter, $\sigma_{s} L_{X}$

$\phi \quad$ Porosity

$\varphi \quad$ Scattering phase function

$w \quad$ Weighting constant

\section{Subscripts}

$b \quad$ Black body

B Bottom

$e \quad$ Exit of the porous matrix

g Gas

$i \quad$ Inlet of the porous matrix

$p \quad$ Porous

T Top

\section{Superscripts}

$m \quad$ Outgoing radiation direction

$m^{\prime} \quad$ Incoming radiation direction

$+\quad$ Upstream direction

- Downstream direction

\section{References}

1. Takeno, T., Sato, K., and Hase, K. "A theoretical study on an excess enthalpy flame", Proceedings of 18th Int. Symposium on Combustion, Waterloo, pp. 465-72 (1981).

2. Echigo, R., Yoshizawa, Y., Hanamura, K., and
Tomimura, T. "Analytical and experimental studies on radiative propagation in porous media with internal heat generation", Proceedings of 8 th Int. Heat Transfer Conf., pp. 827-832 (1986).

3. Yoshida, H., Yung, J.H., Echigo, R., and Tomimura, T. "Transient characteristics of combined conduction, convection and radiation heat transfer in porous media", Int. J. Heat Mass Transfer, 33(5), pp. 847 - 857 (1990).

4. Sathe, S. and Tong, T. "A numerical analysis of heat transfer and combustion in porous radiant burners", Int. J. Heat Mass Transfer, 33, pp. 1331-1338 (1990).

5. Sathe, S., Kulkarni, M.R., Peck, R.E., and Tong, T.W. "An experimental and theoretical study of porous radiant burner performance", International Symposium on Combustion, pp. 1011-1018 (1991).

6. Christo, F.C. "A parametric analysis of a coupled chemistry-radiation model in porous media", DSTORR-0188 (2000).

7. Brenner, G., Pickenacker, K., Pickenacker, O., Trimis, D., Wawrzinek, K., and Weber, T. "Numerical and experimental investigation of matrix-stabilized methane/air combustion in porous inert media", Int. J. Combustion Flame, 123, pp. 201-213 (2000).

8. Talukdar, P., Mishra, S.C., Trimis, D., and Durst, F. "Heat transfer characteristics of a porous radiant burner under the influence of a 2-D radiation field", $J$. Quantitative Spectroscopy \& Radiative Transfer, 84, pp. 527-537 (2004).

9. Lari, K. and Gandjalikhan Nassab, S.A. "Transient thermal characteristics of porous radiant burners", Iranian J. of Science \& Technology, Transition B, Engineering, 31, pp. 407-420 (2007).

10. Farzaneh, M., Ebrahimi, R., Shams, M., and Shafiey, M. "Numerical simulation of thermal performance of a porous burner", Chemical Engineering and Processing: Process Intensification, 48, pp. 623-632 (2009).

11. Keshtkar, M.M. and Gandjalikhan Nassab, S.A. "Theoretical analysis of porous radiant burners under 2-D radiation field using discrete ordinates method", Int. $J$. Quantitative Spectroscopy \& Radiative Transfer, 110, pp. 1894-1907 (2009).

12. Jahanshahi Javaran, E., Gandjalikhan Nassab, S.A., and Jafari, S. "Thermal analysis of a 2-D heat recovery system using porous media including lattice Boltzmann simulation of fluid flow", International Journal of Thermal Sciences, 49(6), pp. 1031-1041 (2010).

13. Chandra, N. and Nakamura, Y. "Modeling and asymptotic analysis of combustion of solid fuel deposited over an inert porous medium", J. Thermal Science and Technology, 12, pp. 723-739 (2012). 
14. Alazmi, B. and Vafai, K. "Constant wall heat flux boundary conditions in porous media under local thermal non-equilibrium conditions", Int. J. Heat Mass Transfer, 45, pp. 3071-3087 (2002).

15. Sigel, R. and Howell, J., Thermal Radiation Heat Transfer, Taylor \& Francis (2002).

16. Mital, R., Gore, J.P., and Viskant, R. "Measurement of extinction coefficient and single scattering albedo of reticulated porous ceramic at high temperatures", 30th AIAA Thermodynamics Conference, June 19-22, San Diego (1995).

17. Escobedo, F. and Viljoen, H.J. "Modeling of porous radiant burners with large extinction coefficients", Canadian J. of Chemical Engineering, 72, pp. 805-814 (1994).

\section{Biographies}

Hoda Shabani Nejad is a PhD student in Mechanical Engineering in the Faculty of Engineering, Shahid Bahonar University, Kerman, Iran. Her MSc thesis is about radiation effect on porous radiant burner. Her research interest includes radiation heat transfer, porous media, and combustion.

Seyed Abdolreza Gandjalikhan Nassab is a Professor in Mechanical Engineering in the Faculty of Engineering, Shahid Bahonar University, Kerman, Iran. He received his $\mathrm{PhD}$ degree from Shiraz University, Shiraz, Iran in 1999. He has many articles in various fields, especially in radiation heat transfer.

Ebrahim Jahanshahi Javaran is an Assistant Professor of Mechanical Engineering at Graduate University of Advanced Technology, Kerman, Iran. He received his BSc, MSc, and $\mathrm{PhD}$ degrees from Shahid Bahonar University of Kerman, Iran in the field of Mechanical Engineering. He is now working on lattice Boltzmann method, solar energy, and desalination systems. He has some publications in the field of radiative heat transfer, rheology of particle suspensions, and solar energy. 